\title{
A Percepção da Inovação Tecnológica em Serviços e a Fidelização de Clientes: uma análise do setor de serviços bancários
}

The Perception of Technological Innovation in Services and a Customer Loyalty: an analysis of the banking sector

Djalma Silva Guimarães Júnior'1, Ricardo José Araújo Silva², Carlos Henrique Michels de Sant'anna ${ }^{1}$, José Everaldo Vilela Martins Júnior ${ }^{1}$, Fagner José Coutinho de Melo ${ }^{1}$

'Universidade de Pernambuco, Pernambuco, Brasil.

${ }^{2}$ União de Ensino Superior de Campina Grande, Paraíba, Brasil.

\section{N F O A R T I GO}

Palavras-chave:

Percepção da inovação, Inovação tecnológica em serviços,

Fidelização de clientes, Bancos.

\section{A R T ICLE IN F O}

\section{Keywords:}

Perception of innovation, Technological innovation in services, Customer loyalty, Banks.

\begin{abstract}
RESUMO
Este artigo tem por objetivo investigar quais são os fatores que afetam a percepção da inovação no serviço bancário, e consequentemente influencia no processo de fidelização do cliente. Para tanto foi utilizada uma modelagem de equações estruturais a partir de 348 questionários aplicados com usuários de serviços bancários, e os resultados apontam que o núcleo do serviço, a tecnologia do serviço e as práticas de gestão significativos para uma percepção positiva da inovação, bem como foi possível uma percepção inovadora favorece a fidelização de clientes a organização. Tais resultados são úteis para a evolução das políticas de melhoria contínua nos serviços e processos alinhados aos anseios dos clientes que possibilitem a agregação de valor. Esta pesquisa contribui com o avanço da literatura de inovação tecnológica em serviços e abre uma possibilidade de exploração do papel da inovação na fidelização de clientes nos mais variados ramos do setor de serviço, de forma a apresentar características específicas de diferentes segmentos.

A B S T R A C T

This paper aims to investigate the factors that affect the perception of innovation in banking service, and consequently influence the customer loyalty process. For this purpose, a structural equation modeling was used based on 348 questionnaires applied to users of banking services, and the results show that the core of the service, the technology of the service and the management practices considered for a positive perception of innovation, as well as a possible innovative perception favors the loyalty of customers to the organization. These results are useful for the evolution of policies for continuous improvement in services and processes that are adequate to the needs of customers that enable the addition of value. This research contributes to the advancement of the literature on technological innovation in services and opens the possibility of exploring the role of innovation in customer loyalty in the most varied branches of the service sector, to present specific characteristics of different segments.
\end{abstract}




\author{
Correspondência para autores: \\ djalma.guimaraes@upe.br (Guimarães JR, D.S.) (ORCID: 0000-0002-4539-8492), \\ araujomail@gmail.com (Silva, R.J.A.) (ORCID: 0000-0002-9019-1933), \\ carlos.santanna@upe.br (de Sant'anna, C.H.M.) (ORCID: 0000-0002-0046-5225), \\ everaldovilela182@gmail.com (Martins JR, J.E.V.) (ORCID: 0000-0002-4179-6960), \\ fagnercoutinhomelo@gmail.com (de Melo,F.J.C.) (ORCID: 0000-0002-0550-5177).
}

\title{
1. Introdução
}

O setor de serviços já representa a maior parcela da riqueza nacional produzida na maioria dos países (AQUINO et al., 2018). Não por acaso é cada vez mais frequente que empresas de outros setores da economia ofereçam soluções de serviços. O segmento industrial, por exemplo, revitalizou sua posição competitiva de forma a dar maior ênfase no serviço ao cliente, incrementado suas receitas a partir de soluções que combinam produtos e serviços de forma a satisfazer as necessidades dos clientes (AQUINO et al., 2019; RAFAEL et al., 2020).

Pesquisas mostram que a receita nominal de serviços vem aumentando conforme dados da Pesquisa Mensal de Serviço em 2018 do Instituto Brasileiro de Geografia e Estatística (IBGE, 2018). Em variação acumulada dos últimos 12 meses, a receita nominal de serviços cresceu 2,4\% em junho de 2018. Tal contexto, endossa o papel crucial do setor de serviços para a economia de qualquer nação, e a inovação tecnológica deve ser um elemento dinamizador deste segmento. Da mesma forma que a indústria busca inovar continuamente em seus produtos, não deve ser diferente no setor de serviços no qual soluções devem ser constantemente desenvolvidas (COLIN, 2019).

Para Thakur e Hale (2013) e Park e Kim (2020) as empresas de serviços necessitam renovar seus processos e oferta para se manterem competitivas. Pois a partir do momento no qual os consumidores são expostos a um novo serviço, é iniciado um processo de avaliação que envolve o desejo de consumir, aceitação ou rejeição emocional, questões de uso e decisão de adoção (ZOLFAGHARIAN; PASWAN, 2008; SANTOS et al., 2020). Caso a empresa não forneça os requisitos adequados para o consumidor ela pode perder o consumidor e posição de mercado. Uma base de clientes fiéis e satisfeitos amplia o volume de venda das empresas (GWINNER; GREMLER; BITNER,1998; CARMONA et al., 2018).

Logo, é estratégico analisar os fatores determinantes da percepção da inovação tecnológica em serviços, bem como verificar o potencial desta para a fidelização de clientes. Entender os fatores que afetam a percepção da inovação tecnológica pode apoiar o direcionamento de esforços mais acurados da empresa em suas iniciativas inovadoras, agregando o máximo de satisfação aos seus consumidores. Tendo em vista a dificuldade de desenvolvimento de práticas de gestão de inovação que empresa brasileira historicamente tem convivido (DE OLIVEIRA BOTELHO; LASSO; MAINARDES 2015; DE ALMEIDA et al., 2020; MELO; MEDEIROS, 2021).

Sendo assim, cabe o questionamento: quais os fatores determinantes da percepção da inovação tecnológica no setor de serviço bancário como potencial para a fidelização de clientes? Com intuito de responder este problema de pesquisa, este artigo tem por objetivo investigar quais são os fatores que afetam a percepção da inovação no serviço bancário, e consequentemente influencia no processo de fidelização do 
cliente.

Desta forma, esta pesquisa pretende contribuir com a literatura de inovação tecnológica em serviços ao investigar quais são os fatores que afetam a percepção da inovação no serviço bancário, e como tal percepção inovadora influencia a continuidade do ciclo de compra a organização, ou seja, a fidelização, por meio da validação do modelo teórico proposto por Zolfagharian e Paswan (2008) utilizando o modelo de equações estruturais. Poucos são os estudos desenvolvidos no setor, com a temática em questão, o que difere este artigo de outros existentes. Atualmente o setor bancário possui uma gama de bancos o que torna o mercado mais competitivo, obrigando com que as organizações ofereças serviços com taxas mais baixas ou equivalentes aos dos outros bancos para fidelizar seus clientes. Segundo o Poder 360 no terceiro trimestre de 2021, os quatro maiores bancos do Brasil (Banco do Brasil, Itaú, Bradesco e Santander) obtiveram lucro superior a R \$ 23 bilhões. Tal conhecimento é essencial no segmento, tendo em vista a ampliação da concorrência do setor e a mudança no comportamento dos clientes sobretudo a partir do crescimento das fintechs no país.

Na sessão a seguir é apresentada uma fundamentação teórica de inovação tecnológica em serviços, bem como a relação entre a inovação e a fidelização, e é apresentado o modelo conceitual investigado; em seguida são delineados os procedimentos metodológicos da pesquisa, resultados, implicações práticas e considerações acerca da pesquisa.

\section{Fundamentação teórica}

Nesta seção, são apresentados os principais conceitos que nortearão a pesquisa. Os principais conceitos estão relacionados a inovação tecnológica em serviços, inovação e a fidelização e o modelo conceitual investigado.

\subsection{Inovação tecnológica em serviços}

A gestão da inovação para o desenvolvimento e aprimoramento contínuo dos produtos é a capacidade chave para atingir os upgrades (avanços) necessários para adentrar na cadeia de valor global econômica. A inovação e o progresso tecnológico corroboram para a ampliação da produtividade e competitividade das organizações, bem como para o desenvolvimento econômico, contribuindo assim para a melhoria das condições de vida de uma sociedade (BARANANO, 2005).

Os objetivos da gestão da inovação nas organizações devem priorizar a capacidade de prover valor ao consumidor final e a capacidade de melhorar os processos continuamente (KAPLINSKY; MORRIS, 2015; CALÁBRIA et al., 2018). Tais objetivos podem enfrentar barreiras, tais como a falta de recursos humanos qualificados e a omissão na comunicação externa da organização com os agentes geradores de conhecimento (universidades e centros de pesquisa) (BARANANO, 2005).

Kaplinsky e Morris (2015) afirmam que os "upgrades" na cadeia de valor estão associados a práticas de inovação nas organizações. A criação de novos produtos, processos e gestão nas indústrias agregam valor a produtos/serviços, ou seja, o upgrade em suas atividades possibilita a competição em uma cadeia de maior valor. Humphrey e Schmitz (2000) partilham desta ideia e chamam a inovação de upgrading, consistindo em as organizações realizarem atividades de modo mais eficiente, agregando valor aos seus processos, fazendo bens através de métodos mais sofisticados. 
No entanto, os recursos investidos visando a melhoria das práticas de inovação em uma organização, muitas vezes podem não fornecer os resultados esperados, em virtude da dificuldade de gerir os aspectos intangíveis do processo, ou seja, dificuldades de gestão da inovação.

Para Baranano (2005) a inovação pode ser potencializada em uma organização a partir dos seguintes fatores: 1 - criação de canais de comunicação fluídos, a criação de inovações depende de vários tipos de conhecimentos que estão distribuídos entre as várias áreas funcionais da organização; 2 - envolvimento dos clientes no processo de inovação, a empresa inovadora deve ser capaz de identificar as necessidades atuais e latentes dos clientes; 3 - apoio da alta gestão da organização; 4 - disponibilidade de recursos humanos qualificados, mudanças e melhorias podem ser induzidas na organização através de colaboradores qualificados, que valorizam a constante aquisição de conhecimentos; e 5 - criação e manutenção de uma estrutura organizacional flexível.

Para a Organização para Cooperação e Desenvolvimento Econômico (OCDE) (MANUAL DE OSLO, 2005) a inovação em serviços consiste no lançamento ou melhoramento significativo de um bem/serviço, processo, método de marketing, metodologia organizacional em práticas comerciais, de trabalho ou relações externas. A inovação nos serviços gera valor para clientes, funcionários, empresários, parceiros de alianças, e comunidade através da oferta de serviços novos/melhorados, processos ou modelos de negócios (OSTROM et al., 2010). A inovação em serviços ocorre em múltiplos escopos, tais como: ciência, tecnologia, processo, necessidade dos clientes, gerenciamento de marcas e internacionalização (CHAE, 2012)

Alguns equívocos no desenvolvimento da pesquisa em inovação nos serviços estão relacionados a sua visão. Boa parte das pesquisas se concentram em uma visão interior da organização e considera a inovação apenas como algo novo para a empresa, negligenciando o desempenho financeiro e o valor gerado para o cliente (SNYDER et al., 2016). Outros problemas na pesquisa de inovação em serviços estão no viés de materialidade, muitos estudos ignoram os aspectos imateriais do serviço, levando a medições imprecisas do impacto econômico e desempenho no setor de serviços (GALLOUJ; SAVONA, 2009).

No entanto, alguns estudos têm orientado uma abordagem no intuito de superar os gargalos da pesquisa em inovação. Ngo e O'Cass (2013) classificam a inovação em duas categorias: capacidade de inovação técnica, que compreende o desenvolvimento de novos serviços, operações de serviço e tecnologia; e capacidades de inovação não técnicas que dizem respeito a ações gerenciais, comerciais e de marketing. Sendo dada pouca atenção a esta última na literatura.

A observação da perspectiva do consumidor na ocasião de sua experiência de serviço pode e deve ser medida, do mesmo, modo que a inovação em produtos é validada através de pesquisas com consumidores, degustações etc., no setor de serviços a presença do consumidor é fundamental para a orientação de esforços de inovação na organização para o cliente.

Hussain, Konar e Ali (2016) apresentam uma proposta para mensuração da performance da inovação em serviços, com base em aspectos relacionados a fatores relacionados a gestão de pessoal dentro da organização, compartilhamento do conhecimento entre a equipe e cultura da equipe do trabalho. Outra proposta para a mensuração da percepção da inovação foi desenvolvida por Zolfagharian e Paswan (2008), os quais investigaram se os consumidores são capazes de discernir as novidades incorporadas em elementos do serviços; o estudo fez uso de uma construção multidimensional e considerou aspectos tangíveis e intangíveis 
do serviço e apontou que os consumidores foram capazes de discernir as seguintes dimensões da inovação nos serviços de academias de ginástica e oficinas mecânicas: instalações interiores, administração, instalações exteriores, funcionários, tecnologia, capacidade de resposta e núcleo do serviço.

As setes dimensões levantadas por Zolfagharian e Paswan (2008), foram validadas no trabalho desenvolvido por Santos et al. (2010), que visou avaliar a inovação na perspectiva do cliente no ambiente dos serviços dos correios e salões de beleza. Os autores utilizaram a análise multivariada no conjunto de 241 dados. Os autores concluíram que as dimensões de instalações exteriores, funcionários, responsividade e instalações interiores são as percebidas como inovativas para os clientes.

O estudo realizado por Kim et al. (2018) visou identificar a percepção inovativa do cliente de restaurante para proposição de uma escala multidimensional. A escala proposta para o setor de restaurante destacou as dimensões núcleo de serviço, tecnologia e responsividade e que na nova escala foram classificadas como menu, serviço baseado em tecnologia, inovação experiencial e promocional.

Keiningham et al. (2019) buscaram verificar a relação entre inovação e autenticidade utilizando uma estrutura conceitual. Os resultados mostraram que a relação entre inovação e autenticidade pode ser mensurada a partir de dois constructos e que essa relação é crucial para o processo de tomada de decisão estratégia que impactam diretamente na fidelidade de lealdade dos clientes. Os autores destacaram que as dimensões que mais significativas levando em consideração tal relação são instalações interiores, instalações exteriores e responsividade.

Silva et al. (2020) desenvolveram um trabalho com intuito de avaliar a percepção de inovação e se essa inovação influencia na fidelização de clientes do serviço de varejo de vestiário. Os autores utilizaram um modelo de regressão linear múltipla num conjunto de 290 questionários. Os resultados mostraram que as dimensões infraestrutura interna da loja, das práticas de gestão da organização e da responsividade do serviço são as que possuem maior intensidade de gerar inovação.

Guimarães Junior et al. (2021) desenvolveram um estudo com intuito de avaliar como o consumidor percebe a inovação e como esta afeta a fidelização em serviços. Os autores utilizaram modelagem de equações estruturais em um compêndio de 1.218 questionários em quatro diferentes segmentos do setor de serviços. Os resultados mostraram que as dimensões gestão e pessoas e inovação percebida foram as consideradas importante para agregar a perspectiva inovadora do serviço. Apesar da apresentação dos artigos ligados a temática, nenhum artigo relacionado a investigar quais são os fatores que afetam a percepção da inovação no serviço bancário, e consequentemente influencia no processo de fidelização do cliente foram encontrados na literatura.

\subsection{Percepção de inovação e fidelização de clientes}

A cada dia os clientes têm se tornado mais exigentes em virtude de suas experiências de serviços, bem como o maior acesso à informação. O que torna cada vez mais desafiador para as organizações a fidelização dos clientes de serviços. O sucesso de um serviço inovador depende da capacidade dos gerentes em combinar o valor que os consumidores procuram no serviço e os incentivos que a empresa recebe para inovar (LENGNICK-HALL, 1992). Ao introduzir inovações a empresa cria oportunidades de marketing, ou seja, laços da empresa com os clientes, contribuindo para uma reputação positiva, que se reflete na fidelidade 
do cliente (CHUN, 2005).

A inovação é o fator chave para o bom relacionamento da organização com o cliente e para a ampliação do potencial de fidelização de clientes, Ruiz-Molina, Gil-Saura e Servera-Francés (2017) identificaram que empresas com práticas inovadoras possuem maior potencial de desenvolver um bom relacionamento e fidelizar clientes, quando comparado a empresas não inovadoras.

A inovação contribui para a percepção de valor no serviço, o consumidor compara os benefícios e os custos oferecidos pela empresa, com os custos e benefícios oferecidos pelos concorrentes, a empresa obtém vantagem competitiva quando é superior em termos de valor para o cliente, trazendo inovações no serviço, processo, ou processo de gestão (YAŞLIOĞLU; ÇALIŞKAN; ŞAP, 2013). As habilidades inovadoras são práticas fundamentais para a formação de valor (HJALAGER, 2010; FLINT LARSSON; GAMMELGAARD, 2008).

Quando se tratar de fidelização de cliente Payne (2006) afirma que manter um cliente antigo tende a ser mais barato que conquistar novos clientes. Para Kottler et al. (2021) as organizações necessitam investir recursos de tempo e financeiro em atributos que impactam diretamente na fidelidade dos clientes.

Autores como Kumar et al. (2013) defendem que o processo de recompra/fidelidade do cliente só acontecerá se o cliente se sentir satisfeito com o serviço prestado pela última vez, e essa satisfação está ligada aos atributos deste serviço prestado. Já Oliver (1999) afirma que o cliente passa por quatro estágios para efetivar uma recompra, são elas: o estágio cognitivo, o estágio afetivo, o estágio conativo e o estágio de ação. Apesar de todo o processo conceitual de recompra/fidelização alguns consumidores compram produtos e serviços sem a intenção real de desenvolver o sentimento de fidelidade. Por fim, Silva et al. (2020) conceituam o processo de fidelização como uma estratégia organizacional focado no cliente por meio de ações de inovação de longo prazo. Na próxima seção é apresentado o modelo conceitual que visa investigar a percepção da inovação no serviço com a fidelização do cliente.

\subsection{Modelo conceitual}

Com base nos construtos presentes na literatura que associam a percepção da inovação tecnológica com fatores tangíveis e intangíveis do serviço, este estudo utiliza os fatores apresentados por Zolfagharian e Paswan (2008):

- Instalações interiores: que consiste na infraestrutura interna da empresa prestadora de serviço;

- Administração: atividades relacionadas indiretamente ao serviço e relacionadas a gestão da empresa;

- Instalações exteriores: relaciona-se a infraestrutura externa do prédio da empresa prestadora de serviço;

- Funcionários, trata da maneira pela qual os funcionários identificam e oferecem serviços adequados para resolver problemas do cliente;

- Tecnologia: corresponde as máquinas e equipamentos utilizados no serviço;

- Capacidade de resposta: é a disponibilidade para prestar o serviço, customização do serviço e a comunicação como o consumidor; 
- Núcleo do serviço: atividades que constituem o serviço em si, ou seja, que são executadas quando o cliente adquire determinado serviço.

A pesquisa também investiga a relação da percepção da inovação no serviço com a fidelização do cliente, conforme apresentado na Figura 1. 
Figura 1: Modelo conceitual.

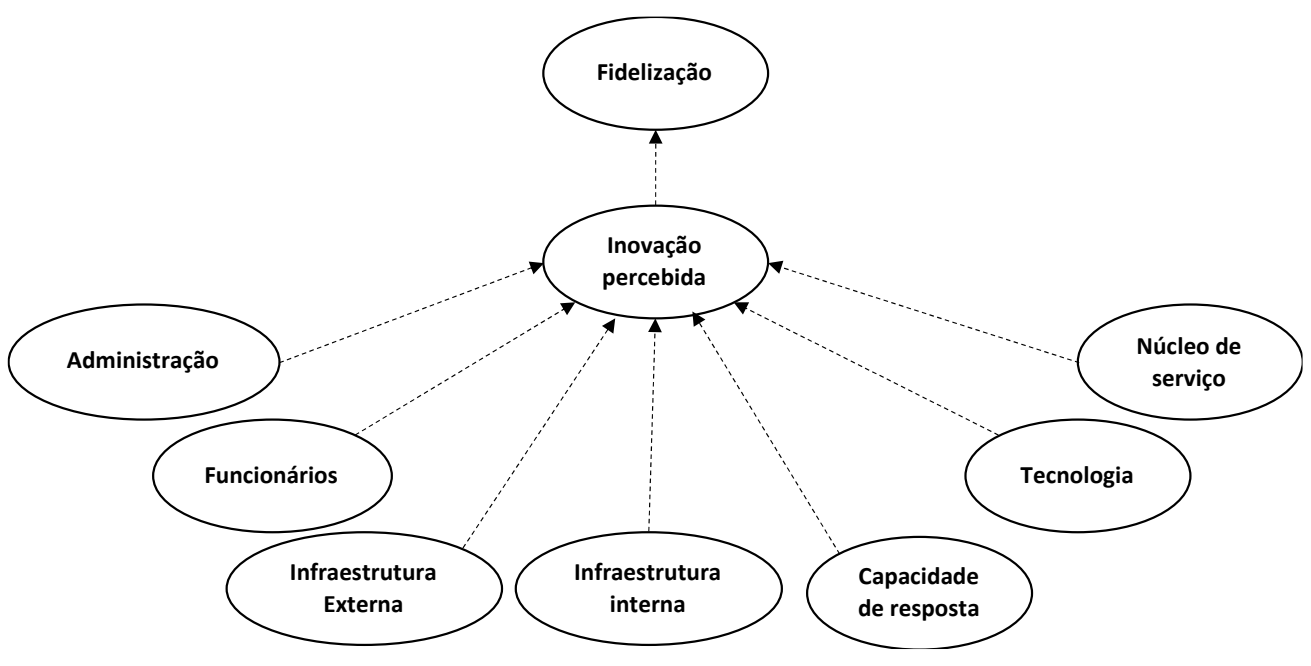

Fonte: Os autores.

\section{Procedimentos metodológicos}

No que diz respeito à forma de abordagem do problema, os dados serão coletados a partir do ambiente natural e analisados sob o enfoque indutivo; as percepções dos clientes obtidas através de questionários serão a base para a análise estatística, com vistas a validação de modelos teóricos, caracterizando assim a pesquisa como quantitativa.

Para a coleta de dados foi utilizada a pesquisa do tipo levantamento, procede-se com a solicitação de informações a um grupo significativo de indivíduos acerca do problema de pesquisa proposto para, mediante análise quantitativa, se obterem as conclusões correspondentes da amostra de dados coletada (VERGARA, 2013).

A etapa de interpretação dos dados, segundo Lakatos e Marconi (2007), corresponde a uma das principais partes da pesquisa, pois é a esta altura que são transcritos os resultados sob a forma de evidências para a confirmação ou não confirmação das hipóteses investigadas. A análise das informações será realizada de forma quantitativa, por meio de modelagem de equações estruturais.

Para o desenvolvimento da pesquisa foram seguidas as seguintes etapas: a) construção do instrumento de coleta de dados, b) validação do instrumento de coleta de dados através de entrevistas com 15 consumidores de serviços bancários a fim de verificar o entendimento das questões propostas no questionário, c) coleta de dados, d) construção do modelo de equações estruturais e análise das saídas do modelo.

O modelo de equações estruturais utiliza as variáveis latentes propostas no modelo conceitual: administração (Adm), funcionários (Func), instalações exteriores (Inext), instalações interiores (Inint), capacidade de resposta (Resp), tecnologia (Tec), e núcleo do serviço (Nser). As variáveis observáveis determinantes das variáveis latentes estão contidas no Anexo I. Bem como as variáveis determinantes dos construtos latentes inovação percebida e fidelidade.

Para realização da pesquisa foram aplicados 348 questionários em usuários de serviços bancários 
residentes no estado de Pernambuco, Brasil no ano de 2017. As entrevistas foram presenciais e os respondentes deveriam necessariamente ter utilizado algum serviço bancário nos dois últimos meses antecedentes a pesquisa, foi utilizado o método de amostragem por conveniência.

\section{Resultados}

O perfil dos respondentes da pesquisa é apresentado na Tabela 1. O grupo etário mais frequente ocorre entre indivíduos de até 25 anos. Também se verifica uma maior proporção de mulheres na pesquisa. Pessoas com ensino médio completo e superior incompleto, representam $64 \%$ dos respondentes. A renda mais frequente ocorre nos grupos de rendimento com até dois salários-mínimos, faixa que representa $81 \%$ do total. O uso pouco frequente do serviço bancário em um mês é outra característica, mesmo com as facilidades apresentadas pela tecnologia de informação e comunicação, $68 \%$ dos entrevistados usam o serviço entre uma e duas vezes no mês, o canal de comunicação preferido ainda é o ambiente físico, a agência.

Tabela 1 - Perfil da Amostra.

\begin{tabular}{|c|c|c|c|}
\hline Característica & Categorias & Ocorrência & $\%$ \\
\hline \multirow{4}{*}{ Idade } & $18-25$ & 153 & 0,44 \\
\hline & $26-35$ & 52 & 0,15 \\
\hline & $36-45$ & 81 & 0,23 \\
\hline & $46-65$ & 52 & 0,15 \\
\hline \multirow{3}{*}{ Sexo } & $66+$ & 10 & 0,03 \\
\hline & Fem & 220 & 0,63 \\
\hline & Mas & 128 & 0,37 \\
\hline \multirow{7}{*}{ Escolaridade } & Fund. Incompleto & 35 & 0,1 \\
\hline & Fund. Completo & 11 & 0,03 \\
\hline & Méd. Incompleto & 16 & 0,05 \\
\hline & Méd. Completo & 110 & 0,32 \\
\hline & Sup. Incompleto & 113 & 0,32 \\
\hline & Sup. Completo & 41 & 0,12 \\
\hline & Pós-Graduação & 22 & 0,06 \\
\hline \multirow{4}{*}{$\begin{array}{l}\text { Renda familiar } \\
\text { (salários mínimos) }\end{array}$} & 1 & 12 & 0,03 \\
\hline & 2-Jan & 272 & 0,78 \\
\hline & 4-Mar & 50 & 0,14 \\
\hline & $5+$ & 14 & 0,04 \\
\hline \multirow{4}{*}{$\begin{array}{l}\text { Frequência de uso } \\
\qquad \text { (mensal) }\end{array}$} & $1-2$ vezes & 238 & 0,68 \\
\hline & $3-5$ vezes & 69 & 0,2 \\
\hline & 6-9 vezes & 17 & 0,05 \\
\hline & $10+$ vezes & 24 & 0,07 \\
\hline
\end{tabular}




\begin{tabular}{cccc} 
& Agência & 237 & 0,68 \\
Canal de & Aplicativo & 78 & 0,22 \\
atendimento & Internet Banking & 27 & 0,08 \\
& Telefone & 6 & 0,02 \\
\hline
\end{tabular}

Fonte: Os autores.

Os dados obtidos a partir dos questionários foram submetidos a um modelo de equações estruturais, construído no software JASP, conforme modelo conceitual proposto na Figura 1. Primeiramente foi verificada a condição de normalidade dos dados, a qual é condição fundamental para a SEM, tal normalidade foi analisada através da assimetria e da curtose. Os valores da assimetria observados em todas as variáveis apresentaram-se valores absolutos inferiores a 3 e a curtose inferiores a 10, atendendo assim as recomendações da literatura, conforme Kline (1998). Desta forma, apesar de nenhuma variável poder se considerada normal, os desvios nos dados não terão efeito sobre a análise a ser realizada.

Na primeira modelagem proposta (Figura 1) as dimensões funcionários (Func), instalações exteriores (Inext), instalações interiores (Inint), capacidade de resposta (Resp) não apresentaram estatísticas significantes, coeficiente padronizado inferior a 0,2 e valor crítico inferior a 1,96. Bem como o ajuste do modelo proposto apresentou um patamar não aceitável, $\mathrm{CFI}=0,601$ e RMSEA=0,090.

Desta forma, o modelo conceitual apresentado na Figura 1 foi reespecificado, considerando apenas as variáveis latentes núcleo do serviço $(\beta=0,857)$, tecnologia $(\beta=0,643)$ e administração $(\beta=0,258)$, apresentaram relações válidas com as variáveis observáveis (valor crítico superior a 1,96 e p-valor inferior a 0,05). Assim, pode-se assumir que tais construtos latentes são representativos do que se propõe a medir conforme Zolfagharian e Paswan (2008). A variável latente inovação percebida no serviço $(\beta=0,475)$ também foi validada pelas suas variáveis observáveis. Os resultados são apresentados na Tabela 2.

Tabela 2 - Modelo proposto.

\begin{tabular}{|c|c|c|c|c|c|}
\hline Variáveis latentes & Relacionamentos & Estimativa & Valor Crítico & $\mathbf{P}$ & Resultado \\
\hline \multirow{6}{*}{ Administração } & $\mathrm{Adm} 03 \leftarrow \mathrm{Adm}$ & 1 & 2,997 & 0,003 & Suportado \\
\hline & $\mathrm{Adm} 02 \leftarrow \mathrm{Adm}$ & 0,965 & 8,863 & $* * *$ & Suportado \\
\hline & $\mathrm{Adm} 01 \leftarrow \mathrm{Adm}$ & 0,728 & 6,935 & $* * *$ & Suportado \\
\hline & $\mathrm{Adm} 04 \leftarrow$ Adm & 0,685 & 7,116 & $* * *$ & Suportado \\
\hline & $\mathrm{Adm} 05 \leftarrow \mathrm{Adm}$ & 0,795 & 7,45 & $* * *$ & Suportado \\
\hline & $\mathrm{Adm} 06 \leftarrow \mathrm{Adm}$ & 0,9 & 8,508 & $* * *$ & Suportado \\
\hline \multirow{3}{*}{ Tecnologia } & $\mathrm{Tec} 01 \leftarrow \mathrm{Tec}$ & 1 & & & Suportado \\
\hline & $\mathrm{Tec} 02 \leftarrow \mathrm{Tec}$ & 1,141 & 7,895 & $* * *$ & Suportado \\
\hline & $\mathrm{Tec} 03 \leftarrow \mathrm{Tec}$ & 1,316 & 7,185 & $* * *$ & Suportado \\
\hline \multirow{4}{*}{ Núcleo do serviço } & Nser01 $\leftarrow$ Nser & 1 & & & Suportado \\
\hline & Nser02 $\leftarrow$ Nser & 0,786 & 7,566 & $* * *$ & Suportado \\
\hline & Nser03 $\leftarrow$ Nser & 0,908 & 8,238 & $* * *$ & Suportado \\
\hline & Nser04 $\leftarrow$ Nser & 1,218 & 9,468 & $* * *$ & Suportado \\
\hline
\end{tabular}




\begin{tabular}{llllll} 
& Inov $\leftarrow$ Adm & 0,258 & 6,678 & $* * *$ & Suportado \\
Inovação Percebida & Inov $\leftarrow$ Tec & 0,643 & 5,736 & $* * *$ & Suportado \\
& Inov $\leftarrow$ Nser & 0,857 & 8,989 & $* * *$ & Suportado \\
& Fid01 $\leftarrow$ Fid & 1 & & & Suportado \\
\multirow{2}{*}{ Fidelização } & Fid02 $\leftarrow$ Fid & 1,127 & 11,58 & $* * *$ & Suportado \\
& Fid03 $\leftarrow$ Fid & 1,179 & 12,598 & $* * *$ & Suportado \\
\hline Fidelização e satisfação & Fid $\leftarrow$ Inov & 0,475 & 6,678 & $* * *$ & Suportado \\
\hline
\end{tabular}

Fonte: Os autores.

As dimensões núcleo do serviço, tecnologia e administração validadas neste artigo estão em consonância com os achados Kim et al. (2018) e Guimarães Junior et al. (2021), apesar de ter sido aplicados em setores de serviço diferentes.

As atividades que são executadas enquanto o cliente recebe o serviço, contribuem fortemente para o desenvolvimento de uma percepção inovadora do serviço. A pesquisa considera as seguintes variáveis como componentes do núcleo do serviço: a disponibilidade de serviços através dos caixas, site e aplicativos, o modo pelo qual os funcionários oferecem instruções para a realização de operações a organização e aparência da agência, caixas, site e aplicativos.

O uso de tecnologias no serviço também apresenta um impacto forte na percepção da inovação. Os seguintes requisitos tecnológicos são significativos para influenciar a percepção dos clientes: estado dos equipamentos (computadores, terminais de autoatendimento e guichês); a maneira pela qual são organizadas as filas e distribuídas as senhas; e o uso de tecnologias que facilitem os pagamentos.

Os que os fatores relacionados as práticas de gestão da organização, apesar de significantes, contribuem com menor intensidade para inovação percebida. As práticas de gestão indutoras de inovação consideradas na pesquisa são: a maneira pela qual a instituição divulga os seus produtos, a forma de interação com os clientes, o modo de entretenimento durante a espera, a política de preços e a capacidade de identificação de necessidades.

A partir da significância da relação entre a inovação percebida e da fidelização, pode-se confirmar a hipótese central da pesquisa, ou seja, a inovação percebida no serviço influencia a fidelização de clientes, conforme Ruiz-Molina, Gil-Saura e Servera-Francés (2017). Desta forma, quanto mais elevada percepção da inovação através dos fatores núcleo do serviço, tecnologia e administração, maior a tendência da repetição do uso do serviço bancário por parte do cliente.

As relações apresentadas na Tabela 2 foram consideradas válidas, conforme atestado pelo valor crítico superior a 1,96 em todas as estimativas. Bem como, os indicadores de ajuste do modelo proposto atendem ao indicado na literatura, $\mathrm{CMIN} / \mathrm{DF}=2,296$ (indicação $<5$ ), $\mathrm{CFI}=0,922$ (indicação >0,9), e RMSEA $=0,601$ (indicação $>0,05 ;<0,08$ ). No que diz respeito a validade da escala utilizada para a mensuração dos construtos latentes pode-se verificar valores de confiabilidade composta superiores a 0,7 .

\section{Conclusão}

A literatura sugere que a inovação tecnológica em serviços pode e deve ser mensurada, de forma a aprimorar o desempenho das organizações. Entretanto, ainda são escassos na literatura as investigações acerca 
do tema. Esta pesquisa atua para minimizar tal escassez ao concluir que os aspectos relacionados ao núcleo do serviço prestado, tecnologia e administração atuam para a formação de uma percepção inovadora do serviço da instituição bancária. Tal percepção amplia o potencial de fidelização do cliente.

A pesquisa confirmou a relação proposta por Ruiz-Molina, Gil-Saura e Servera-Francés (2017) entre a inovação e a fidelização de clientes, bem como, apontou para a oportunidade de ações de melhoria no segmento bancário mais efetivas para a satisfação e fidelização, que são: 1 - ações inovadoras nas operações bancárias propriamente ditas, desenvolvimento de novas formas de realização de saques, transferências, atualizações cadastrais, etc., possuem potencial de agregar valor ao serviço e fidelizar os clientes. Ao se considerar que o uso do serviço bancário na amostra ainda é majoritariamente presencial, pode ser, relevante para tal público a geração de incentivos para o uso de aplicativos de celular e internet banking; 2 - outro fator, que pode corroborar para ampliação da percepção inovadora são os recursos de tecnologia, a aparência e facilidade de uso dos terminais, os equipamentos para organização do serviço interno, senhas e painéis de chamadas, bem como a facilidade de canais disponibilizados para a realização das transações; 3 - o terceiro aspecto do serviço significativo para percepção inovadora e fidelização ao banco, são práticas de gestão, as quais dizem respeito a necessidade de melhorias na maneira na qual a empresa apresenta seus serviços, entretém nos momentos de espera, flexibilidade nas formas de pagamentos, e precificação dos serviços.

Muitas iniciativas relacionadas a inovação em serviços não conseguem lograr êxito em virtude de uma abordagem voltada para aspectos internos, deixando de lado a participação do feedback dos clientes neste processo. Assim este trabalho corrobora para a ampliação da participação dos clientes no processo de desenvolvimento de estratégias de inovação em serviços, bem como, representa um avanço na compreensão do papel da inovação sob a ótica do cliente para os serviços bancários, em um ambiente cada vez mais intensivo no uso de tecnologias digitais.

Desta forma, considera-se que a pesquisa de inovação em serviços, a partir da perspectiva do usuário, pode auxiliar organizações do segmento no aprimoramento de seus processos internos e de comunicação com o público de forma a agregar valor aos serviços prestados corroborando com Baranano (2005). É importante ressaltar que a pesquisa e o entendimento de pontos críticos para a inovação em serviços, como visto nos resultados deste trabalho, fornecem uma margem de aprimoramento dos serviços que só é viabilizada ao se considerar o papel central do consumidor.

A busca individual de competitividade através da inovação gera um upgrade na cadeia de valor, o qual contribui para o desenvolvimento de um ciclo virtuoso na economia a partir da ampliação da produtividade, a qual contribui para o desenvolvimento econômico, que por sua vez, melhora as condições de vida da sociedade. Assim, o desenvolvimento contínuo de processos inovadores gera um efeito que vai além da organização e de seus clientes, e se espraia por toda a sociedade.

São fatores limitantes da pesquisa a escassez de trabalhos relacionados a inovação em serviços bancários na perspectiva do consumidor, o tamanho e o tipo da amostragem propostas nesta pesquisa. Devese ressaltar ainda que a transformação recente do setor promovida pelas fintechs é um fator determinante na experiência do serviço que precisa ser melhor compreendido.

No entanto, a pesquisa abre uma possibilidade de exploração do papel da inovação na fidelização de clientes nos mais variados ramos do setor de serviço, de forma a apresentar características específicas de 
diferentes segmentos. A pesquisa ainda pode servir como ponto de partida para a análise da percepção dos consumidores sobre os novos serviços fornecidos a partir da transformação digital em andamento no sistema bancário. 


\section{Referências}

AQUINO, J.T.; MELO, F.J.C.; JERÔNIMO, T.B.; MEDEIROS, D.D. Evaluation of Quality in Public Transport Services: The Use of Quality Dimensions as an Input for Fuzzy TOPSIS. International Journal of Fuzzy Systems, v. 21, n. 2, p. 176-193, 2019.

AQUINO, J.T.; SOUZA, J.V.; SILVA, V.C.L.; JERONIMO, T.B.; MELO, F.J.C. Factors that influence the quality of services provided by the bus rapid transit system: a look for user's perception. Benchmarking, $\mathrm{v}$. 25, n. 3. p. 4035-4057, 2018.

BARAÑANO, A.M. Gestão da inovação tecnológica: estudo de cinco PMEs portuguesas. Revista Brasileira de Inovação, v. 4, n. 1, p. 57-96, 2005.

CALÁBRIA, F.; MELO, F.J.C.; ALBUQUERQUE, A.P.G.; JERONIMO, T.B.; MEDEIROS, D.D. Changing the training paradigm for learning: A model of human capital development. Energy \& Environment, v. 29, n. 2, p. 1455-1481, 2018.

CARMONA, L. J. M.; TOMELIN, J.; DANI, A. C.; HEIN, N. Efeito da intensidade tecnológica na relação entre o investimento em inovação e o desempenho organizacional de setores industriais. Brazilian Journal of Management \& Innovation, v. 5, n. 2, p. 84-106, 2018.

CHAE, B.K. An evolutionary framework for service innovation: Insights of complexity theory for service science. International journal of production economics, v. 135, n. 2, pp. 813-822, 2012.

CHUN, R. Corporate reputation: Meaning and measurement. International Journal of Management Reviews, v. 7, n. 2, p. 91-109, 2005.

COLIN, G. Artificial Intelligence and Japan's Fifth Generation: The Information Society, Neoliberalism, and Alternative Modernities. Pacific Historical Review, v. 88, n. 4, p. 619-658, 2019.

DE ALMEIDA, N.A.S.; RODAS, S.E.R.; MARQUES, W.M.R. Investimento em pesquisa e inovação tecnológica: um estudo de caso para o Brasil. Revista Estudo \& Debate, v. 27, n. 1, p. 7-28, 2020.

DE OLIVEIRA BOTELHO, D.A.; LASSO, S.V.; MAINARDES, E.W. Fatores de inovação das empresas capixabas. Revista Brasileira de Gestão e Inovação, v. 2, n. 2, p. 26-53, 2015.

FLINT, D.J.; LARSSON, E.; GAMMELGAARD, B. Exploring processes for customer value insights, supply chain learning and innovation: an international study. Journal of Business Logistics, v. 29, n. 1, p. 257 $281,2008$.

GALLOUJ, F.; SAVONA, M. Innovation in services: a review of the debate and a research agenda. Journal of evolutionary economics, v. 19, n. 2, p. 149, 2009.

GUIMARÃES JUNIOR, D.S.; MELO, F.J.C.; ALBUQUERQUE, A.P.G.; SOBRAL, E.F.M. Development of loyalty through innovation perceived in services. International journal of services and operations management, $v$. ahead-of-print, $n$. ahead-of-print, pp. 1-20, 2021.

GWINNER, K.P.; GREMLER, D.D.; BITNER, M.J. Relational benefits in services industries: the customer's perspective. Journal of the academy of marketing science, v. 26, n. 2, p. 101-114, 1998.

HJALAGER, A.M. A review of innovation research in tourism. Tourism management, v. 31, n. 1, p. 1-12, 2010.

HUMPHREY, J.; SCHMITZ, H. Governance and upgrading: linking industrial cluster and global value chain research. Institute of Development Studies, 2000.

HUSSAIN, K.; KONAR, R.; ALI, F. Measuring service innovation performance through team culture and knowledge sharing behaviour in hotel services: a PLS approach. Procedia-Social and Behavioral Sciences, v. 224, n. 15, p. 35-43, 2016.

IBGE - Instituto Brasileiro de Geografia e Estatística - Pesquisa Mensal de Serviços IBGE. Índice da Receita Nominal de Serviços. Abril 2018 A junho 2018. Disponível em: www.ibge.gov.br/estatisticasnovoportal, Acessado em: 31 agosto, 2018.

KAPLINSKY, R; MORRIS, M. Thinning and thickening: productive sector policies in the era of global value chains. The European Journal of Development Research, v. 28, n. 4, p. 625-645, 2016. 
KEININGHAM, T. L. et al. Creating innovation that drives authenticity. Journal of Service Management, $v$ 30, n. 3, p. 369-391, 2019.

KIM, E.; TANG, L. R.; BOSSELMAN, R. Measuring customer perceptions of restaurant innovativeness: Developing and validating a scale. International Journal of Hospitality Management, v. 74, p. 85-98, 2018.

KLINE, R.B., Principles and practice os structural equation modeling. New York, The Guildford Press, 1998.

KOTLER, P.; KARTAJAYA, H.; SETIAWAN, I. Marketing 5.0: Technology for humanity. John Wiley \& Sons, 2021.

KUMAR, V.; DALLA POZZA, I.; GANESH, J. Revisiting the satisfaction-loyalty relationship: empirical generalizations and directions for future research. Journal of retailing, v. 89, n. 3, p. 246-262, 2013.

LAKATOS, E.M.; MARCONI, M.A. Fundamentos de metodologia científica. 5. reimp. São Paulo: Atlas, p. 310, 2007.

LENGNICK-HALL, C.A. Innovation, and competitive advantage: What we know and what we need to learn. Journal of management, v. 18, n. 2, p. 399-429, 1992.

LIN, C-Y. How does perceived retail service innovativeness affect retail patronage intentions? Creativity and Innovation Management, v. 28, n. 4, p. 519-532, 2019.

MANUAL DE OSLO. Manual de Oslo: diretrizes para coleta e interpretação de dados sobre inovação. Brasília: OCDE, Finep, 2005.

MELO, F.J.C.; MEDEIROS, D.D. Applying interpretive structural modeling to analyze the fundamental concepts of the management excellence model guided by the risk-based thinking of ISO 9001: 2015. Human and Ecological Risk Assessment: An International Journal, v. 27, n.02, p. 742-772, 2021.

NGO, L.V.; O'CASS, A. Innovation and business success: The mediating role of customer participation. Journal of Business Research, v. 66, n. 8, p. 1134-1142, 2013.

OLIVER, R. L. Whence consumer loyalty? The Journal of Marketing, p. 33-44, 1999.

OSTROM, A.L. et al. Moving forward and making a difference: research priorities for the science of service. Journal of service research, v. 13, n. 1, p. 4-36, 2010.

PARK, C.Y., KIM, J. Education, Skill Training, and Lifelong Learning in the Era of Technological Revolution. Asian Development Bank Economics Working Paper Series, (606), 2020.

PAYNE, A. Handbook of CRM: Achieving Excellence in Customer Relationship Management. Oxford: Butterworth-Heinemann Publications; Elsevier, 2006.

PODER 360. (2021). Lucro dos 4 maiores bancos do país alcança R\$ 23 bilhões no $3^{\circ}$ trimestre. Disponível em: https://www.poder360.com.br/economia/lucro-dos-4-maiores-bancos-do-pais-alcanca-r-23-bilhoes-no3o-trimestre/. Acessado em: 01 de dezembro de 2021.

RAFAEL, L. D.; JAIONE, G. E.; CRISTINA, L.; IBON, S.L. An Industry 4.0 maturity model for machine tool companies. Technological Forecasting and Social Change, v. 159, p. 120203, 2020.

RUIZ-MOLINA, M.E.; GIL-SAURA, I.; SERVERA-FRANCES, D. Innovation as a key to strengthen the effect of relationship benefits on loyalty in retailing. Journal of Services Marketing, v. 31, n. 2, p. 131-141, 2017.

SANTOS, H. O.; ALVES, J. L. S.; DE MELO, F. J. C.; DE MEDEIROS, D. D. An approach to implement cleaner production in services: Integrating quality management process. Journal of Cleaner Production, v. 246, p. 118985, 2020.

SANTOS, A. M.; FREITAS, A. A. F; ASSIS, A; SIMÕES, D. A percepção da Inovação Pelo Consumidor no Contexto de Serviços. Vitoria/ES, 2010. Disponível em: < http://www.anpad.org.br/admin/pdf/simposio159.pdf>. Acesso em: 16 ago. 2020.

SILVA, B. R.; GUIMARÃES JUNIOR, D. S.; ARRUDA, D. R.; MELO, F. J. C. Inovação no varejo de vestuário: percepção do cliente e fidelização. Revista Opara, v. 10, n. 02, p.71-84, 2020. 
SNYDER, H. et al. Identifying categories of service innovation: A review and synthesis of the literature.

Journal of Business Research, v. 69, n. 7, p. 2401-2408, 2016.

THAKUR, R.; HALE, D.. Service innovation: A comparative study of US and Indian service firms. Journal of Business Research, v. 66, n. 8, p. 1108-1123, 2013.

VERGARA, S.C. Projetos e relatórios de pesquisa. São Paulo: Atlas, 2006.

YAŞLIOĞLU, M.; ÇALIŞKAN, B.Ö.Ö.; ŞAP, Ö. O papel da inovação e da qualidade percebida do serviço na criação de valor para o cliente: um estudo com funcionários de um estabelecimento de call center.

Procedia-Social and Behavioral Sciences, v. 99, p. 629-635, 2013.

ZOLFAGHARIAN, M.; PASWAN, A. Do consumers discern innovations in service elements? Journal of Services Marketing, v. 22, n. 5, p. 338-352, 2008.

\section{Anexo I}

Este questionário faz parte de uma pesquisa desenvolvida pela Universidade de Pernambuco (UPE). Os

RESULTADOS desta pesquisa serão CONFIDENCIAIS. Por favor, leia atentamente o questionário e responda as perguntas.

\section{QUESTIONÁRIO - PARTE I}

1) Idade: anos

2) Sexo:Feminino ( ) Masculino( )

3) Qual o seu nível de escolaridade?

( ) Ensino fundamental incompleto

( ) Ensino fundamental completo

( ) Ensino médio incompleto

( ) Ensino médio completo

( ) Ensino superior incompleto

( ) Ensino superior completo

( ) Pós-graduação

4) a) Em qual cidade você mora?

b) Em qual bairro você mora?

5) Qual é a sua renda?

6) Frequência de uso? (Vezes por mês)

7) Canal mais utilizado?

( ) Agência física ( ) Aplicativo de celular ( ) Internet Banking (Computador) ( ) Telefone

\section{PARTE II - QUESTÕES FUNCIONAIS}

Por favor, marque um X na resposta que melhor traduz seus sentimentos a respeito dos serviços prestados pelo $\underline{\text { Banco }}$ no qual você possui conta.

Considere: 1 - Nenhum pouco inovador e 5 - Extremamente inovador. 


\begin{tabular}{|c|c|c|c|c|c|c|}
\hline $\begin{array}{c}\text { Variável } \\
\text { latente }\end{array}$ & ITENS & 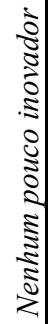 & 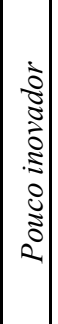 & $\mid$ & 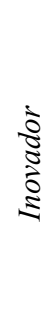 & 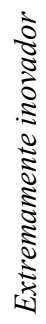 \\
\hline Adm01 & A forma como o banco divulga seus produtos é & 1 & 2 & 3 & 4 & 5 \\
\hline Adm02 & A forma como o banco interage com os clientes é & 1 & 2 & 3 & 4 & 5 \\
\hline Adm03 & O modo como entretém os clientes durante a espera do serviço & 1 & 2 & 3 & 4 & 5 \\
\hline Adm04 & As formas de pagamento disponíveis são & 1 & 2 & 3 & 4 & 5 \\
\hline Adm05 & As tarifas (taxas) para diferentes serviços são & 1 & 2 & 3 & 4 & 5 \\
\hline Adm06 & A maneira como identifica o que os clientes querem é & 1 & 2 & 3 & 4 & 5 \\
\hline Func01 & A forma como os funcionários tentam responder aos questionamentos dos clientes é & 1 & 2 & 3 & 4 & 5 \\
\hline Func02 & A forma como os funcionários ajudam a solucionar os problemas dos clientes é & 1 & 2 & 3 & 4 & 5 \\
\hline Func03 & A forma como disponibiliza funcionários para orientar os clientes sobre produtos e serviços é & 1 & 2 & 3 & 4 & 5 \\
\hline Func04 & O vestuário utilizado pelos funcionários é & 1 & 2 & 3 & 4 & 5 \\
\hline Inext01 & A iluminação fora do prédio é & 1 & 2 & 3 & 4 & 5 \\
\hline Inext02 & O design exterior do prédio é & 1 & 2 & 3 & 4 & 5 \\
\hline Inext03 & A comunicação visual externa & 1 & 2 & 3 & 4 & 5 \\
\hline Inext04 & A forma de acesso ao interior do prédio é & 1 & 2 & 3 & 4 & 5 \\
\hline Inint01 & O sistema de ventilação (climatização) é & 1 & 2 & 3 & 4 & 5 \\
\hline Inint02 & A segurança é & 1 & 2 & 3 & 4 & 5 \\
\hline Inint03 & A aparência das paredes, piso e teto é & 1 & 2 & 3 & 4 & 5 \\
\hline Inint04 & A comunicação visual interna (placas de orientação) é & 1 & 2 & 3 & 4 & 5 \\
\hline Inint05 & O uso de itens como móveis e outras mobílias é & 1 & 2 & 3 & 4 & 5 \\
\hline Inint06 & A disposição dos móveis e equipamentos é & 1 & 2 & 3 & 4 & 5 \\
\hline Inint08 & A iluminação interior é & 1 & 2 & 3 & 4 & 5 \\
\hline Resp01 & Os dias de funcionamento são & 1 & 2 & 3 & 4 & 5 \\
\hline Resp02 & O horário de funcionamento é & 1 & 2 & 3 & 4 & 5 \\
\hline Resp03 & A comunicação com os clientes é & 1 & 2 & 3 & 4 & 5 \\
\hline Resp04 & O modo como a empresa personaliza os serviços para diferentes clientes é & 1 & 2 & 3 & 4 & 5 \\
\hline Resp05 & Os canais disponibilizados para reclamações, elogios e sugestões são & 1 & 2 & 3 & 4 & 5 \\
\hline Tecn 01 & Os caixas eletrônicos, site e aplicativo são & 1 & 2 & 3 & 4 & 5 \\
\hline Tecn02 & A forma de organização de fila (por exemplo, fila única, distribuição de senhas, etc.) & 1 & 2 & 3 & 4 & 5 \\
\hline Tecn03 & A tecnologia utilizada para facilitar o pagamento pelos serviços prestados & 1 & 2 & 3 & 4 & 5 \\
\hline NSer01 & A maneira como são disponibilizados os caixas, site e aplicativo é & 1 & 2 & 3 & 4 & 5 \\
\hline Nser02 & O modo c/ os funcionários oferecem instruções sobre como proceder para realizar o serviço é & 1 & 2 & 3 & 4 & 5 \\
\hline Nser03 & A maneira de organização dos caixas, site e aplicativo é & 1 & 2 & 3 & 4 & 5 \\
\hline
\end{tabular}




\begin{tabular}{|l|l|l|l|l|l|l|}
\hline Nser04 & As máquinas e equipamentos da agência, site e aplicativo são & 1 & 2 & 3 & 4 & 5 \\
\hline
\end{tabular}

\section{PARTE III - Avaliação Global}

Assinale a afirmativa que melhor representa a sua última experiência com os serviços prestados pelo banco que você mais utiliza:

\begin{tabular}{|c|c|c|c|c|c|}
\hline ITENS & 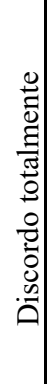 & 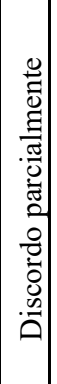 & $\begin{array}{l}0 \\
0 \\
0 \\
0 \\
: 0 \\
0 \\
0 \\
0 \\
0 \\
0 \\
0 \\
0 \\
0 \\
0 \\
0 \\
0 \\
0 \\
z\end{array}$ & 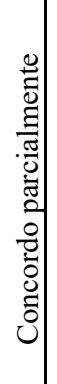 & 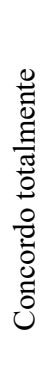 \\
\hline 1. Considero excelente o serviço recebido no banco. & 1 & 2 & 3 & 4 & 5 \\
\hline 2. O serviço que recebi está entre os melhores do mercado. & 1 & 2 & 3 & 4 & 5 \\
\hline 3. O serviço que recebo me fideliza a empresa. & 1 & 2 & 3 & 4 & 5 \\
\hline 4. O serviço me satisfaz de forma que não considero buscar um serviço concorrente. & 1 & 2 & 3 & 4 & 5 \\
\hline 5. Pretendo continuar utilizando os serviços da organização. & 1 & 2 & 3 & 4 & 5 \\
\hline 6. Falo bem dos serviços da empresa para outras pessoas. & 1 & 2 & 3 & 4 & 5 \\
\hline
\end{tabular}

Em uma escala de 1 a 5, como você classificaria a SUA SATISFAÇÃO COM A QUALIDADE GERAL dos serviços prestados pelo Banco na última vez?

\begin{tabular}{|c|l|l|l|l|l|}
\hline & $\mathbf{1}$ & $\mathbf{2}$ & $\mathbf{3}$ & $\mathbf{4}$ & $\mathbf{5}$ \\
\hline Agência & & & & & \\
\hline Site & & & & & \\
\hline Aplicativo & & & & & \\
\hline
\end{tabular}

